\title{
Clinical outcome and toxicity for immunotherapy treatment in metastatic cancer patients
}

\author{
Kin Sang Lau, Ronald Liu, Cheuk Cheuk Wong, Wai Kwan Steven Siu, Kwok Keung Yuen \\ Department of Clinical Oncology, Queen Mary Hospital, University of Hong Kong, Hong Kong, China \\ Contributions: (I) Conception and design: KS Lau, WKS Siu, KK Yuen; (II) Administrative support: All authors; (III) Provision of study materials \\ or patients: KS Lau, R Liu; (IV) Collection and assembly of data: KS Lau, R Liu, CC Wong; (V) Data analysis and interpretation: KS Lau; (VI) \\ Manuscript writing: All authors; (VII) Final approval of manuscript: All authors. \\ Correspondence to: Dr. Kin Sang Lau. Queen Mary Hospital, Department of Clinical Oncology, 1/F, Professorial Block, Queen Mary Hospital, 102 \\ Pokfulam Road, Hong Kong, China. Email: LKS417@ha.org.hk.
}

Background: Immunotherapy (IO) is known to improve survival and outcome in various types of solid tumours. However, nonspecific activation of the immune system also affects various organ systems leading to the immune-related adverse events (irAEs). Systematic reviews of IO trials show that the actual incidence of irAEs may be higher than expected. Little is known about the impact of these irAEs on patients' clinical outcome, palliative care (PC) needs and hospice service use.

Methods: This is a single centre, retrospective review study of metastatic cancer patients between June 2016 to June 2017 who consecutively received immune checkpoint inhibitors with anti-PD1 in our institution. The computerized medical record, body weight chart, blood test results and in-patient assessment records were reviewed. The study was approved by the Institutional Review Board of the University of Hong Kong/ Hospital Authority Hong Kong West Cluster and conducted in compliance with the Declaration of Helsinki. Results: Fifty patients received immune checkpoint inhibitors with anti-PD1 consecutively between June 2016 to June 2017 were retrospectively reviewed. The median age was 64 years old (range: 22 to 87 years old). Thirty-three of them were male (66\%) patients. Twenty-five patients $(50 \%)$ experienced any grade irAE. Ten patients (20\%) experienced grade III/IV irAE among which 7 patients (14\%) discontinued IO treatment permanently and 2 patients (4\%) died due to grade III/IV toxicity. The development of grade III/ IV irAE required in-patient management, with a median duration of hospitalization of 6.5 days (range: 1 to 38 days). The response rate was 36\% vs. 4\% ( $\mathrm{P}=0.01$ ), median PFS (15.8 vs. 6.2 months, $\mathrm{P}=0.26)$, median OS (21.0 vs. 12.9 months, $\mathrm{P}=0.05)$ for patients with or without irAEs, respectively. The occurrence of any grade irAE was associated with a trend of improved overall survival (OS) on IO $(\mathrm{P}=0.05)$. Five patients $(10 \%)$ developed hyper-progressive disease and received only one course of treatment before they died. Only 2 patients (4\%) developed pseudo-progressive disease during treatment. Thirty-five mortalities (70\%) occurred at the time of assessment of the study, of which 18 patients (36\%) received PC consultations and 12 patients (24\%) received hospice care before they passed away.

Conclusions: Our study underscored the need for enhanced selection criteria to identify patient subgroups which benefit most from IO, and the need to involve PC and hospice services early for those non-responders or unlikely responders. Patient education and a dedicated multi-disciplinary team approach is needed to identify and treat irAE timely to prevent severe morbidities and mortalities.

Keywords: Immunotherapy (IO); toxicity; palliative

Submitted Apr 15, 2019. Accepted for publication Sep 26, 2019.

doi: 10.21037/apm.2019.10.03

View this article at: http://dx.doi.org/10.21037/apm.2019.10.03 


\section{Introduction}

Immunotherapy (IO) has revolutionized the treatment paradigms for a broad spectrum of solid malignancies. Immune checkpoint inhibitor is the class of IO approved for use in many different types of solid tumours which consists of anti-PD1 or anti-PDL1 and the CTLA-4 inhibitors. Cancer cells harbour mechanisms to evade normal immune surveillance and immune checkpoint inhibitors prevent these cancer cells from evading the immune T-cell attacks. Because immune checkpoint inhibitors rely on immune reactivation to combat cancer cells, this drug class mechanism may lead to the loss of immune tolerance and result in a wide range of toxicities called immune-related adverse events (irAEs).

Toxicities are in general more common with antiCTLA4 than anti-PD-1. Toxicities are often subtle in the early phase, and can theoretically occur at any time after a single dose of immune checkpoint inhibitor (1). Although toxicities of IO are quoted as being less common than traditional chemotherapy, they are not uncommon as encountered in clinical practice. If toxicities are left unnoticed, they may progress to life threatening organ failure if not act upon timely.

Anti-PD1 is one of the commonest immune checkpoint inhibitors approved to be in use in various metastatic cancers. Anti-PD1 has been demonstrated to be an effective regimen in metastatic non-small cell lung cancer (2), advanced head and neck cancer (3), genitourinary cancer (4,5), melanoma (6) and other cancers such as gastrointestinal cancer.

Anti-PD1 was shown to be effective in prolonging survival and improving the quality of life of advanced head and neck cancer patients. The Keynote 024 trial showed improved overall survival (OS) and a longer time to deterioration of patient-reported quality of life scores compared with chemotherapy (7). The Checkmate 141 trial showed improvement of median progression-free survival and increase in quality-of-life scores in head-andneck cancers with nivolumab (3). The Keynote 045 trial showed improvement of median OS and health-related quality-of-life scores (7). The Checkmate 214 showed improved OS and improved patient-reported quality of life scores compared with the standard targeted therapy sunitinib for intermediate or poor risk group of renal cell carcinoma (8).

The pattern of response to treatment as well as the pattern of side effects with IO differ from those with traditional cytotoxic chemotherapy and targeted therapy.
The response to IO may take considerably longer to be observed radiologically compared with cytotoxic chemotherapy and targeted therapy. Clinically, patients may experience disease stabilization or even improvement before objective radiologic response takes place. The patients receiving IO may demonstrate a period of disease stabilization or even transient worsening of radiologic lesions before radiologic tumor regression occurs. This phenomenon of transient worsening of radiologic lesions is called pseudo-progressive disease. Some cautions are generally taken by oncologists experienced in IO not to stop IO prematurely so that potential benefit from IO would not be compromised. However, this specific type of delayed response generally does not occur in patients with symptomatic deterioration or worsening performance status. Therefore, continuation of IO beyond progression for patients with apparent clinical deterioration is not recommended. Additionally, there is a distinct phenomenon known as hyper-progressive disease.

The phenomenon of "Pseudo-progressive disease" and "Hyper-progressive disease" are the observed characteristic pattern of response to IO which are class specific to IO $(9,10)$

The incidence of genuine pseudo-progressive disease is in general less than $5 \%$ in solid tumors as reported in the literature (11). Hyper-progressive disease is a phenomenon of extraordinarily rapid tumor progression which confers a worse prognosis to cancer patients (12). Predictive factors of hyper-progressive disease are largely unknown. Some investigators suggested that the risk of hyper-progressive disease may be related to age, previous irradiation and metastatic tumor load (13). Patients who show signs of hyper-progressive disease should stop IO treatment and consider alternative therapy and early palliative care (PC).

Research has been conducted in an attempt to identify clinical parameters and routinely available blood tests to predict survival outcome and toxicities to IO (14) so as to identify patients who would benefit from IO and to avoid the severe immune-related toxicities. The ECOG performance status was found to be an important pretreatment prognostic factor of survival outcome for advanced non-small cell lung cancer (15) and melanoma (16). Any grade irAE was associated with enhanced response rate and OS from nivolumab treatment of melanoma patients (17). Routinely available blood tests including Absolute Neutrophil Count (ANC) at week 6 post-IO (16), neutrophil-to-lymphocyte ratio (NLR) (15), 
Increased relative lymphocyte count (RLC) and relative eosinophil count at baseline (18) were investigated as predictors for IO response and toxicity.

Little is known about the predictive value of the implicated clinical parameters and blood tests for IO response and toxicity. In addition, the impact of these irAEs on patients' clinical outcome, PC needs and hospice service use were seldomly reported (19). There is an unmet need in patient selection criteria for receiving IO from both clinical and laboratory predictor marker for IO response and toxicity. The current trends and timing of integration of PC consultations and hospice services for those non-responders or unlikely responders is another focus of this research.

\section{Methods}

This is a single centre, retrospective review study of metastatic cancer patients between June 2016 to June 2017 who consecutively received immune checkpoint inhibitors with anti-PD1 in our institution. Immune checkpoint inhibitors, pembrolizumab and nivolumab were approved for single agent use in 2016. The computerized medical record, body weight chart, blood test results and in-patient assessment records were reviewed. Patients who were not primarily treated in our institution with no complete medical records, those without regular clinical monitoring or blood tests screening or those without regular computed tomography scan assessment were excluded. Sixty-five patients were identified in the period between June 2016 to June 2017. After exclusion, 50 patients were included in the final analysis in the study.

All patients included in the study has regular imaging assessment with either computed tomography scan or $18-\mathrm{F}$ FDG positron emission tomography computed tomography scan at an interval of 3 months or more frequently as clinically indicated. Baseline and regular blood monitoring scheduled were in accordance to published guideline by the European Society of Medical Oncology.

The overall response rate was evaluated according to the Response Evaluation Criteria in Solid Tumors version 1.1. The median progression free survival (PFS) and OS were estimated using the Kaplan-Meier Method. Toxicity was reported according to the National Cancer Institute Common Terminology Criteria for Adverse Events version 4.0. Patient characteristics and variables of interest for the group with or without PC consultation, and the group with or without irAE occurrence were compared using the Fisher's exact test or Chi-square test as appropriate. The odds ratio of clinical covariates and blood test variables impacting occurrence of grade III/IV irAE were analysed using the logistic regression method. The median PFS and OS of two groups were compared by the log-rank test and hazard ratios of covariates (if found significant by univariate analyses) by the Cox proportional hazard method.

The study was approved by the Institutional Review Board of the University of Hong Kong/Hospital Authority Hong Kong West Cluster (reference number: UW 18678) and conducted in compliance with the Declaration of Helsinki.

\section{Results}

\section{Patient characteristics}

Fifty patients who received immune checkpoint inhibitors with anti-PD1 consecutively between June 2016 to June 2017 were retrospectively reviewed. The median age was 64 years old (range: 22 to 87 years old). Thirty-three of them were male $(66 \%)$ patients. Eighteen patients $(36 \%)$ received Nivolumab and 32 patients received Pembrolizumab (64\%).

Twenty-five patients (50\%) experienced any grade irAE and 10 patients (20\%) experienced grade III/IV irAE.

The response rate was $36 \%$ vs. $4 \%(\mathrm{P}=0.01)$, median $\mathrm{PFS}$ (15.8 vs. 6.2 months, $\mathrm{P}=0.26)$, median OS (21.0 vs. 12.9 months, $\mathrm{P}=0.05$ ) for patients with or without irAEs, respectively.

Five patients (10\%) developed hyper-progressive disease and received only one course of treatment before they died. Only 2 patients (4\%) developed pseudo-progressive disease during treatment. Thirty-five mortalities $(70 \%)$ occurred at the time of assessment of the study, of which 18 patients (36\%) received PC consultations and 12 patients (24\%) received hospice care before they passed away.

Patient characteristics were compared between those with PC consultation and without as shown in Table 1.

\section{Pseudo-progressive vs. hyper-progressive disease in relation to PC and Hospice care}

Five patients (10\%) developed hyper-progressive disease and received only one course of treatment before they died. Only 2 patients (4\%) developed pseudo-progressive disease during treatment. Thirty-five mortalities $(70 \%)$ occurred at the time of assessment of the study, of which 18 patients (36\%) received PC consultations and 12 patients (24\%) received hospice care before they passed away.

Patients were more likely to received PC consultations if 
Table 1 Patient characteristics: PC consultation $(\mathrm{n}=18)$ vs. without PC consultation $(\mathrm{n}=32), \mathrm{n}(\%)$

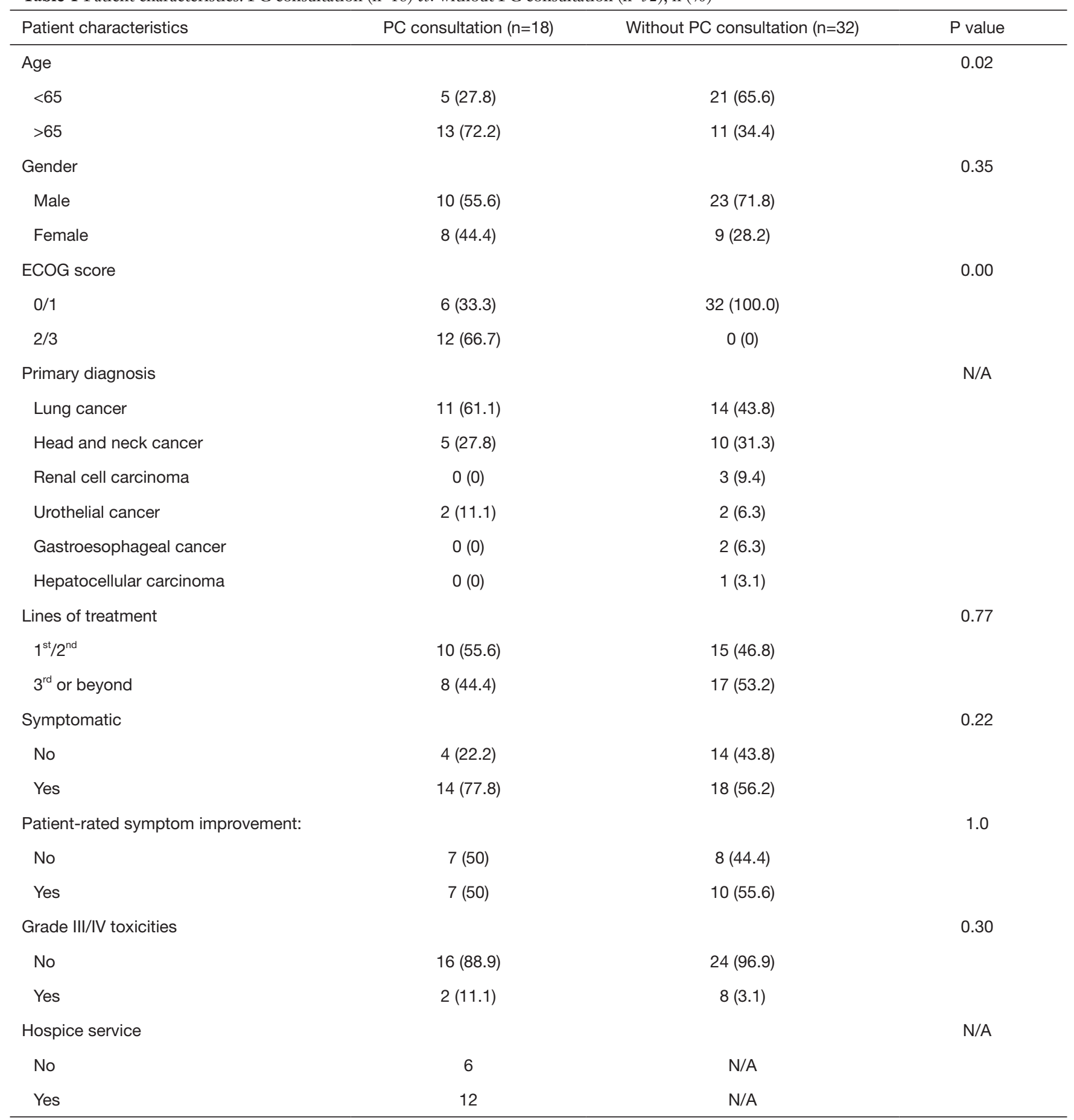

$\mathrm{P}$ value by Fisher Exact test 2-tailed. PC, palliative care. 
Table 2 Comparison of patient characteristics: with irAE $v s$. without irAE, n (\%)

\begin{tabular}{|c|c|c|c|}
\hline Patient characteristics & With irAE & Without irAE & $P$ value \\
\hline Age & & & 1.00 \\
\hline$<65$ & $13[52]$ & $13[52]$ & \\
\hline$\geq 65$ & $12[48]$ & $12[48]$ & \\
\hline Gender & & & 0.23 \\
\hline Male & $19[76]$ & $14[56]$ & \\
\hline Female & $6[24]$ & $11[44]$ & \\
\hline ECOG score & & & 0.74 \\
\hline $0 / 1$ & $20[80]$ & $18[72]$ & \\
\hline $2 / 3$ & $5[20]$ & 7 [28] & \\
\hline Lines of treatment & & & 1.00 \\
\hline $1^{\mathrm{st}} / 2^{\mathrm{nd}}$ & $12[48]$ & $13[52]$ & \\
\hline $3^{\text {rd }}$ or beyond & $13[52]$ & $12[48]$ & \\
\hline \multicolumn{4}{|l|}{ No. of cycles received } \\
\hline $1-5$ cycles & 9 [36] & $14[56]$ & \\
\hline $6-10$ cycles & $6[24]$ & $5[20]$ & \\
\hline 11-20 cycles & $4[16]$ & $4[16]$ & \\
\hline 21-30 cycles & $4[16]$ & $1[4]$ & \\
\hline $31-50$ cycles & $2[8]$ & $1[4]$ & \\
\hline \multicolumn{3}{|c|}{ History of autoimmune disease } & 0.50 \\
\hline Yes & $1[10]$ & $2(2.5)$ & \\
\hline No & 9 [90] & $38(97.5)$ & \\
\hline BW change & $+1.80 \mathrm{~kg}$ & $+0.10 \mathrm{~kg}$ & 0.23 \\
\hline \multicolumn{3}{|c|}{ Response rate on assessment scan at $\leq 3$ months } & 0.01 \\
\hline $\mathrm{SD} / \mathrm{PD}$ & $16[64]$ & $24[96]$ & \\
\hline $\mathrm{CR} / \mathrm{PR}$ & 9 [36] & $1[4]$ & \\
\hline Progression-free survival & 441 days & 174 days & 0.26 \\
\hline Overall survival & 587 days & 362 days & 0.05 \\
\hline
\end{tabular}

$P$ value by Fisher Exact test 2-tailed. irAE, immune-related adverse event.

age $\geq 65$ years old $(\mathrm{P}=0.02)$ or having baseline ECOG status of 2 or above $(\mathrm{P}=0.00)$ as shown in Table 1.

\section{Patient characteristics in relation to any grade irAE}

Age older than or equal to 65 years old $(\mathrm{P}=1.00)$ and male gender $(\mathrm{P}=0.23)$ were not associated with the occurrence of any grade irAE.

Baseline ECOG score $(\mathrm{P}=0.74)$ and the lines of treatment $(\mathrm{P}=1.00)$ were not associated with the occurrence of any grade irAE.

History of autoimmune disease was not associated with any grade $\operatorname{irAE}(\mathrm{P}=0.50)$.

Having a complete response or partial response on early assessment scan was associated with the occurrence of any grade irAE $(\mathrm{P}=0.01)$ as shown in Table 2.

The mean body weight change of patients on IO were not significantly different for those with or without occurrence of irAE.

\section{Grade III/IV irAE, onset, duration and outcomes}

Ten patients (20\%) experienced grade III/IV irAE, as detailed in Table 3. Three patients (6\%) underwent invasive procedures of either biopsy or endoscopy for diagnosis or management of irAE. Eighy patients (16\%) received systemic corticosteroid for at least 1 month. Two patients (4\%) required the addition of intravenous immunoglobulin for management of grade III/ IV irAE. Four patients (8\%) developed a secondary infection and 2 patients (4\%) died as a result. Seven patients (14\%) discontinued IO treatment permanently due to grade III/IV toxicity. The development of grade III/IV irAE required inpatient management, with a median duration of hospitalization of 6.5 days (range: 1 to 38 days).

Three patients $(6 \%)$ had a history of autoimmune disease (Graves' disease) and none of them developed any grade irAE. Four patients (8\%) developed immune-related endocrinopathies in which 3 of them were hypothyroidism and one was severe hypophysitis, admitted for ICU care who was subsequently associated with mortality. Seven patients (14\%) developed immune-related dermatitis in which one of them was severe toxic epidermoid necrolysis leading to ICU stay for over a month. One patient (2\%) developed grade III immune-related nephritis and required ICU stay for haemodialysis. No known risk factors were significantly associated with subsequent development of grade III/IV irAE.

\section{With vs. without occurrence of any-grade irAE}

The response rate was $36 \%$ vs. $4 \%(\mathrm{P}=0.005)$, median PFS (15.8 vs. 6.2 months, $\mathrm{P}=0.26)$, median OS (21.0 vs. 12.9 months, $\mathrm{P}=0.05$ ) for patients with or without irAEs, respectively. Early response to IO on assessment scan at 
Table 3 Summary of Grade III/IV irAE, onset, duration, length of hospitalization and outcomes

\begin{tabular}{|c|c|c|c|c|c|c|c|c|c|}
\hline Gr34irAE & $\begin{array}{l}\text { Onset in } \\
\text { Days }\end{array}$ & $\begin{array}{l}\text { Duration of } \\
\text { irAE in days }\end{array}$ & $\begin{array}{l}\text { Hospitalization } \\
\text { in days }\end{array}$ & $\begin{array}{c}\text { Endoscopy or } \\
\text { Biopsy }\end{array}$ & $\begin{array}{l}\text { Steroid } \\
\text { use }\end{array}$ & IV Ig & Infection & Discontinue $T x$ & Death \\
\hline Hypophysitis & 67 & 21 & 21 & $x$ & $\sqrt{ }$ & $x$ & $x$ & $\sqrt{ }$ & $\sqrt{ }$ \\
\hline Colitis & 110 & 30 & 3 & $x$ & $\sqrt{ }$ & $x$ & $x$ & $x$ & $x$ \\
\hline TENS & 42 & 49 & 38 & $\sqrt{ }$ & $\sqrt{ }$ & $\sqrt{ }$ & $\sqrt{ }$ & $\sqrt{ }$ & $x$ \\
\hline Vomit Mallory Weiss & 225 & 7 & 7 & $\sqrt{ }$ & $x$ & $x$ & $x$ & $x$ & $x$ \\
\hline $\begin{array}{l}\text { Nephritis renal } \\
\text { failure }\end{array}$ & 71 & 30 & 30 & $x$ & $\sqrt{ }$ & $\sqrt{ }$ & $\sqrt{ }$ & $\sqrt{ }$ & $x$ \\
\hline Anaemia & 65 & 21 & 1 & $x$ & $\sqrt{ }$ & $x$ & $x$ & $\sqrt{ }$ & $x$ \\
\hline
\end{tabular}

irAE, immune-related adverse event.

Table 4 Factors impacting early response to anti-PD1 (\%)

\begin{tabular}{lccc}
\hline Covariates & Odds ratio (OR) & $95 \% \mathrm{Cl}$ & $\mathrm{P}$ value \\
\hline Any grade irAE & 13.5 & $1.6-117.1$ & 0.01 \\
Age $\geq 65$ & 0.7 & $0.2-2.7$ & 0.57 \\
ECOG 0/1 & 0.3 & $0.0-2.6$ & 0.27 \\
NLR & 1.1 & $0.9-1.2$ & 0.34 \\
RLC & 0.3 & $0.0-1,039.0$ & 0.76 \\
ELR & 0.7 & $0.8-384.6$ & 0.07
\end{tabular}

$P$ value by univariate logistic regression. irAE, immune-related adverse event.

3 months or earlier was associated with any-grade irAE toxicity $(\mathrm{P}=0.01)$ as shown in Table 4.

\section{Association of blood parameters with toxicity}

None of the blood parameters including NLR $(\mathrm{P}=0.34)$, relative lymphocyte ratio (RLR) $(\mathrm{P}=0.76)$, or eosinophilto-lymphocyte ratio (ELR) $(\mathrm{P}=0.07)$ were significantly associated with toxicity.

There was no difference observed between Pembrolizumab and Nivolumab for the incidence of grade III/IV irAE $(\mathrm{P}=0.30)$ as shown in Table 5 .
Table 5 Comparison of grade I/II irAE: pembrolizumab vs. nivolumab, n (\%)

\begin{tabular}{|c|c|c|c|}
\hline Common irAE & Pembrolizumab & Nivolumab & $P$ value \\
\hline Arthralgia $[n=2(4 \%)]$ & & & 0.53 \\
\hline Yes & $2(6.3)$ & $0(0.0)$ & \\
\hline No & $30(93.7)$ & $18(100.0)$ & \\
\hline Skin rash $[\mathrm{n}=7$ (14\%)] & & & 0.40 \\
\hline Yes & $6(18.8)$ & $1(5.6)$ & \\
\hline No & $26(81.2)$ & $17(94.4)$ & \\
\hline Colitis [n=2 (4\%)] & & & 0.53 \\
\hline Yes & $2(6.3)$ & $0(0)$ & \\
\hline No & $30(93.7)$ & $18(100.0)$ & \\
\hline Hepatitis [n=3 (6\%)] & & & 1.0 \\
\hline Yes & $2(6.3)$ & $1(5.6)$ & \\
\hline No & $30(93.7)$ & $17(94.4)$ & \\
\hline Hypothyroidism [n=3 (6\%) & & & 1.0 \\
\hline Yes & $2(6.3)$ & $1(5.6)$ & \\
\hline No & $30(93.7)$ & $17(94.4)$ & \\
\hline Pneumonitis [n=1 (2\%)] & & & 1.0 \\
\hline Yes & $1(3.1)$ & $0(0.0)$ & \\
\hline No & $31(96.9)$ & $18(100.0)$ & \\
\hline
\end{tabular}




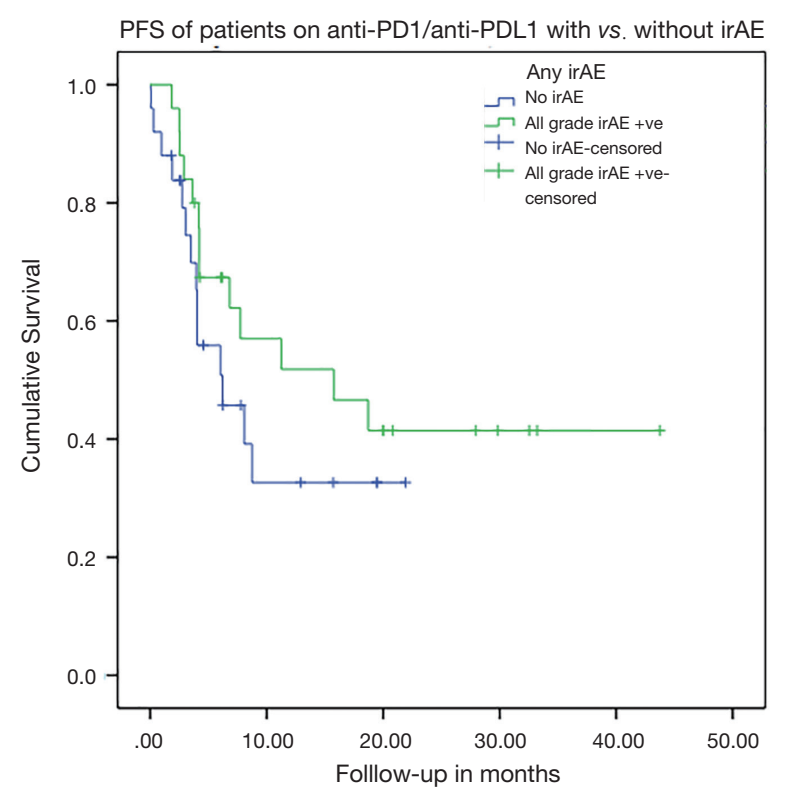

Figure $1 \mathrm{KM}$ curve of PFS survival of patients with $v s$. without any-grade irAE $(\mathrm{P}=0.26)$. irAE, immune-related adverse event.

\section{KM analysis for PFS (log rank test)}

The occurrence of any grade irAE was not associated with a trend of improved PFS on IO $(\mathrm{P}=0.26)$ as shown in Figure 1 .

\section{KM analysis for OS (log rank test)}

The occurrence of any grade irAE was associated with a trend of improved OS on IO $(\mathrm{P}=0.05)$ as shown in Figure 2.

\section{Discussion}

When patients with late-stage cancers run out of other options, IO is considered more often as the last resort. PC needs, end-of-life decisions of patients and hospice services are often introduced late in the disease course. It was observed in clinical practice that a trial of $\mathrm{IO}$ as the last resort is in lieu of the discussions with PC specialists about advance-care planning.

IO brings new hope on efficacious treatment options to patients. This new anti-cancer agent has its pros and cons. In unselected patients, the median response rate to IO is $20 \%$ in this study. Among the responders, symptom improvements occurred in $46.9 \%$. The median incidence rate of grade III/IV toxicities is $20 \%$, among which the median duration of hospitalization was 14.8 days. Invasive procedures for diagnosis and management were required

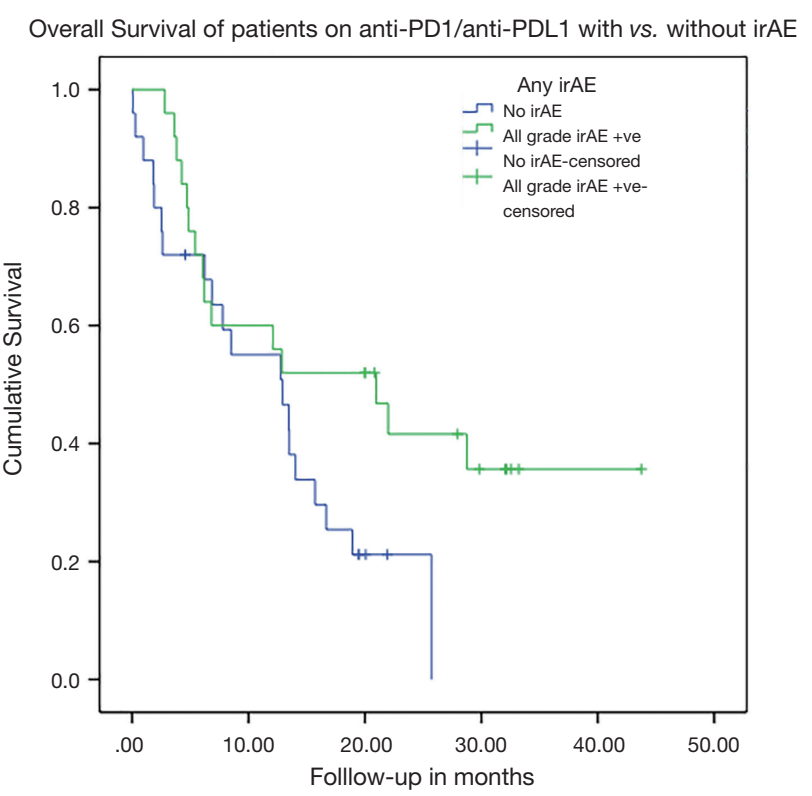

Figure 2 KM curve of OS survival of patients with $v s$. without anygrade irAE $(\mathrm{P}=0.05)$. irAE, immune-related adverse event.

in $30 \%$. High systemic dose of steroid was needed to treat grade III/IV irAE in $80 \%$ which leads to treatment discontinuation and death in $70 \%$ and $10 \%$ of patients respectively.

In this study, younger patients $(<65$ years old $)$ were less likely to receive $\mathrm{PC}$ consultations $(\mathrm{P}=0.02)$. Among patients who received PC consultations, $27.8 \%$ of them was $<65$ years old and $72.2 \%$ was $>65$ years old. Only $24 \%$ of patients died in hospice, except for the longterm responders, the others died in acute hospitals upon emergency admissions.

Earlier PC referral and integration of PC into the care of metastatic cancer patients could bring more timely palliative specialists' counselling and advanced care planning. Emergency admissions could be reduced, especially for patients already receiving late lines of therapy or having deteriorated performance status. When quality-of-life goals and end-of-life issues were addressed at an earlier phase, patient satisfaction could be enhanced and transition to hospice care could be facilitated.

The response rate of IO is generally poor for certain subgroup with specific driver mutation e.g., EGFR mutation in lung cancer (20). However, no single reliable molecular biomarker was identified to predict the response or toxicity from IO. At the moment, PD-L1 level on tumor specimen is the most common biomarker to indicate the use 
of immune checkpoint inhibitor $(21,22)$. However, tumors without PD-L1 staining might still respond to anti-PD1.

Studies of IO toxicity and prediction of response found that the occurrence of irAE was associated with enhanced clinical benefit (23), improved progression-free survival and OS from immune checkpoint inhibitor as reported in the literature (24). In this study, the response rate was $36 \%$ vs. 4\% ( $\mathrm{P}=0.005)$, median PFS (15.8 vs. 6.2 months, $\mathrm{P}=0.26)$, median OS (21.0 vs. 12.9 months, $\mathrm{P}=0.05)$ for patients with or without irAEs, respectively. Early response to IO on assessment scan at 3 months or earlier was associated with any-grade irAE toxicity $(\mathrm{P}=0.01)$.

Absolute neutrophil counts at week 6 after nivolumab treatment has been associated with improved OS in melanoma patients (16). Raised NLR at baseline was associated with increased risk of death and disease progression in metastatic lung cancer patients treated with nivolumab (15). Increased RLC and relative eosinophil count at baseline was associated with improved OS (18). In this study, none of the blood parameters including the postIO difference in absolute leucocyte, neutrophil, lymphocyte, eosinophil, NLR, RLC, or ELR were significantly associated with any-grade or grade III/IV toxicity. Advanced age and ECOG score were not found to be significant predictor of grade III/IV toxicity. The lack of statistical power of this study due to a small sample size is the most likely cause to explain.

The European Society of Medical Oncology (ESMO) and American Society of Clinical Oncology (ASCO) have issued guidelines in the management of immune related adverse effects $(25,26)$. For the diagnosis of immune related thyroiditis causing hypothyroidism, baseline biochemistry tests including TSH, free T4, early morning cortisol, and other routinely available hormones are needed before the commencement of IO. If concomitant adrenal insufficiency is suspected, dynamic functional testing under medical endocrine team supervision is advised. Concomitant hypothyroidism and adrenal insufficiency should raise the suspicion of severe hypophysitis, which would warrant additional testing of pituitary hormones, pituitary MRI scan and consideration of early systemic corticosteroid, electrolyte and hormone replacement (27). For immune related thyroiditis alone, regular thyroxine replacement should be given with regular thyroid function blood checking, and anti-PD1 can be safely continued (28). For immune-related skin toxicity, grading is according to symptoms severity and percentage body surface area involvement. Anti-PD1 should be withheld until skin toxicity resolved to grade I severity, oral antihistamines and topical steroid can be considered. Systemic steroid use should be considered for grade III or above severity. If symptoms failed to improve, apart from systemic steroid, other immune-modulating agents e.g., immunoglobulin should be considered and patients should be managed in collaboration with dermatologist, plastic surgeon in an intensive care unit (ICU) or burn unit. Skin biopsy may be required for difficult diagnosis (25).

For immune-related hepatitis and nephritis, anti-PD1 should be withheld until resolution to grade I severity. If grade II laboratory derangement persists, systemic steroid should be administered. The dose-titration of steroid should be according the published guideline. The dose of steroid should be, in general, tapered over 4 weeks of time to avoid rebound of toxicity. For steroid refractory cases, other immune-modulating agents e.g., mycophenolate mofetil, infliximab. should be considered for the appropriate types of irAE. Supportive transient haemodialysis may be required for immune-related nephritis that recovered over protracted period (26).

Management of irAE requires specialist experienced in $\mathrm{IO}$ toxicities and there should be high level of suspicion that new symptoms are IO-related until proven otherwise, as delayed appropriate management could result in mortality (25).

While discontinuation of IO and the use of corticosteroid can result in resolution of irAEs, long-term sequelae and mortality could occur (29). Patient education and early recognition of symptoms is of paramount importance.

Immune-related pneumonitis was reported to occur in $3-5 \%$ of patients receiving immune checkpoint inhibitors (30). In this study, 1 patient (2\%) developed grade II pneumonitis which resolved with systemic steroid. A plain chest CT scan is the modality of choice for diagnosing immune-related pneumonitis, though the radiologic appearance of the pulmonary toxicity varied between different cohorts. For grade II pneumonitis, prednisolone dose of $1 \mathrm{mg} / \mathrm{kg} /$ day is recommended. For grade III/ IV pneumonitis, prednisolone dose of $2-4 \mathrm{mg} / \mathrm{kg} / \mathrm{day}$ is recommended. Patients who remain without clinical improvement after 72 hours of high-dose corticosteroid are considered steroid-refractory who are immediately fatal within days. In this situation, other immune-modulating agents e.g., IV immunoglobulin or infliximab are often considered with varying degree of success.

One of the rare grade III/IV irAE reported in this study is tuberculous ileitis which is rarely reported. Though the literature on tuberculosis infection (TB caused by 
anti-PD1 antibody treatment is sparse, the possibility of acute TB infection or TB reactivation is a recognized phenomenon (31-33). One less frequently reported grade III/IV irAE reported in this study is severe immunerelated neurologic toxicity. In one recent review article, the incidence of immune-related neurologic toxicity ranges from $1-12 \%$ for patients receiving immune checkpoint inhibitors (34). A subset of patient with this rare toxicity runs a fulminant course with increasing headache and muscle pain, dysphagia, paralysis and eventually death.

IO is not recommended in the end-of-life period as a means to defer PC decisions. The use of IO near the end-of-life period may lead to more ICU care or patients dying in the acute ward. The experience of distressing symptoms and toxicities from IO leading to death could be a devastating suffering to both patients and family, leading to regret for treatment.

The actual rate of ICU usage for patients receiving IO is under reported. Few articles described about ICU usage for grade III/IV toxicities of anti-PD1 in the literature. One recent review article on improving the awareness of intensive care physicians to the severe toxicity from immune checkpoint inhibitors shed some light on the problems (35). Grade III/IV Pneumonitis leading to ICU admission was described in up to $1 \%$ of patients receiving anti-PD1. Severe acute respiratory distress syndrome (ARDS) is the common pathway of development of severe pneumonitis. None of the study included described specific features that distinguish immune-related pneumonitis from other etiologies of pneumonitis, which often posed a dilemma to ICU colleagues. The diagnosis of immunerelated pneumonitis causing ARDS requires a high level of suspicion and remains a diagnosis by exclusion while some patients required the use of lung biopsy (35). According to a recent review article on the impact of another new form of IO called CAR-T cell therapy, at least $15 \%$ of patients in the pivotal clinical trials required ICU admission (36). Immune checkpoint inhibitors are different from CAR-T cell therapy. In our study, 3 patients $(6 \%)$ required ICU support for the management of grade III/IV irAE. Though the incidence of grade III/IV toxicity and ICU usage is lower for immune checkpoint inhibitors, its significance should not be neglected. There is certainly an unmet need in identifying predictors of toxicity from IO so that devastating sufferings can be prevented.

With the emerging research findings on IO biomarkers and predictors, more specific prediction models should be formulated to select the likely responders with low risk of severe toxicities, who would benefit most from this efficacious new treatment strategy.

The preferred approach to the management of metastatic cancer patients follows a multi-disciplinary approach, with early phase-in of care from the palliative specialists (37). In this study, patients with age older than or equal to 65 years old or having baseline ECOG score of 2 or above were more likely referred early for PC consultations. With an early phase-in approach, patients should be referred for PC service irrespective of their age. In particular, for patients receiving IO who suffer from significant treatment-related toxicity or who have no early response to treatment, should be referred early for PC services. An interdisciplinary PC team and a simultaneous care approach are the advocated approach, with some advocating the combined tumor board approach (38). For high-volume centres treating a large number of patients with IO, the combined irAE tumor board approach may facilitate timely inputs from various specialties resulting in a coordinated approach to the management of irAE (39).

There are few limitations of this study. The sample size is small (50 patients), and there is relatively short followup time ( $<3$ years). This may affect the generalizability of results. Most of our patients received IO after the announcement of approval of the drug by the Food and Drug Administration in the United States in 2016, which means recruitment of study subjects before year 2016 is not practical for our current study. The response and likelihood of immune-related toxicity could be related to the dose-intensity of individual immune checkpoint inhibitor received, but the current study was not powered to investigate this dose response relationship. To answer this research question, a pre-planned large prospective study is required.

The study population contains a heterogeneous group of cancer disease from various primary and location of metastases. One systematic review of the tumor-specific pattern of irAEs of immune checkpoint inhibitors suggests that the pattern of irAE can be different between melanoma and non-small cell lung cancer, or between melanoma and renal cell carcinoma (40). However, the pattern of irAE between non-small cell lung cancer and renal cell carcinoma was not shown to be significantly different and that melanoma patients may have a distinct immune response from other cancer types. In this study, 32 patients (64\%) have the diagnosis of non-small cell lung cancer or renal cell carcinoma or urothelial cancer, 15 patients 
(30\%) have the diagnosis of head-and-neck cancer, 2 patients (4\%) have esophago-gastric cancer and 1 patient (2\%) has hepatocellular carcinoma. No melanoma patients were found in this study due to the rarity of disease in this locality. No significant difference in response was observed between tumor types in this study. For assessment of the impact of an intervention on patients' quality of life and symptom improvement, baseline assessment tool such as the Quality of Life at the End of Life scale (QUAL-E) and the Edmonton Symptom Assessment System (ESAS) could be used as commonly employed in clinical trials (41). Future prospective study on the impact of IO on the quality of life, the functional aspect and the symptom improvement of patients receiving PC services or hospice care should made use of these standardized instrument to quantify the impact of interest.

Up to the time of write-up of this study, there is no published study on the impact of immune-related toxicity of immune checkpoint inhibitors on clinical outcomes in metastatic cancer patients in relation to their PC need and hospice use (19). Heightened enthusiasm in the possibility of long-term survival and possibly cure within the vastly developing IO community could partly explained the lack of literature on the palliative aspects on the topic.

\section{Conclusions}

In conclusion, this study underscored the need for enhanced selection criteria to identify patient subgroups who might benefit most from IO, and the need to involve PC and hospice services early for those non-responders or unlikely responders. Patient education and a dedicated multidisciplinary team approach are needed to identify and treat irAE in a timely manner to prevent severe morbidities and mortalities.

\section{Acknowledgments}

Funding: None.

\section{Footnote}

Provenance and Peer Review: This article was commissioned by the Guest Editors (Rebecca Yeung and Tai Chung Lam) for the series "Integrating Palliative Medicine in Oncology Care: The Hong Kong Experience", published in Annals of Palliative Medicine. This article has undergone external peer review.
Conflicts of Interest: All authors have completed the ICMJE uniform disclosure form (available at http://dx.doi. org/10.21037/apm.2019.10.03). The series "Integrating Palliative Medicine in Oncology Care: The Hong Kong Experience" was commissioned by the editorial office without any funding or sponsorship. Dr. KK Yuen received study cost paid to the institution from AstraZeneca. The authors have no other conflicts of interest to declare.

Ethical Statement: The authors are accountable for all aspects of the work in ensuring that questions related to the accuracy or integrity of any part of the work are appropriately investigated and resolved. The study was approved by the Institutional Review Board of the University of Hong Kong/Hospital Authority Hong Kong West Cluster (reference number: UW 18-678) and conducted in compliance with the Declaration of Helsinki (as revised in 2013).

Open Access Statement: This is an Open Access article distributed in accordance with the Creative Commons Attribution-NonCommercial-NoDerivs 4.0 International License (CC BY-NC-ND 4.0), which permits the noncommercial replication and distribution of the article with the strict proviso that no changes or edits are made and the original work is properly cited (including links to both the formal publication through the relevant DOI and the license). See: https://creativecommons.org/licenses/by-nc-nd/4.0/.

\section{References}

1. Michot JM, Bigenwald C, Champiat S, et al. Immunerelated adverse events with immune checkpoint blockade: a comprehensive review. Eur J Cancer 2016;54:139-48.

2. Reck M, Rodriguez-Abreu D, Robinson AG, et al. Pembrolizumab versus Chemotherapy for PD-L1Positive Non-Small-Cell Lung Cancer. N Engl J Med 2016;375:1823-33.

3. Gillison ML, Blumenschein G Jr, Fayette J, et al. CheckMate 141: 1-Year Update and Subgroup Analysis of Nivolumab as First-Line Therapy in Patients with Recurrent/Metastatic Head and Neck Cancer. Oncologist 2018;23:1079-82.

4. Motzer RJ, Tannir NM, McDermott DF, et al. Nivolumab plus Ipilimumab versus Sunitinib in Advanced Renal-Cell Carcinoma. N Engl J Med 2018;378:1277-90.

5. Sharma P, Retz M, Siefker-Radtke A, et al. Nivolumab in metastatic urothelial carcinoma after platinum therapy 
(CheckMate 275): a multicentre, single-arm, phase 2 trial. Lancet Oncol 2017;18:312-22.

6. Larkin J, Chiarion-Sileni V, Gonzalez R, et al. Combined Nivolumab and Ipilimumab or Monotherapy in Untreated Melanoma. N Engl J Med 2015;373:23-34.

7. Vaughn DJ, Bellmunt J, Fradet Y, et al. Health-Related Quality-of-Life Analysis From KEYNOTE-045: A Phase III Study of Pembrolizumab Versus Chemotherapy for Previously Treated Advanced Urothelial Cancer. J Clin Oncol 2018;36:1579-87.

8. Cella D, Grunwald V, Escudier B, et al. Patient-reported outcomes of patients with advanced renal cell carcinoma treated with nivolumab plus ipilimumab versus sunitinib (CheckMate 214): a randomised, phase 3 trial. Lancet Oncol 2019;20:297-310.

9. Wolchok JD, Hoos A, O'Day S, et al. Guidelines for the evaluation of immune therapy activity in solid tumors: immune-related response criteria. Clin Cancer Res 2009; 15:7412-20.

10. Ferrara R, Mezquita L, Texier M, et al. Hyperprogressive Disease in Patients With Advanced Non-Small Cell Lung Cancer Treated With PD-1/PD-L1 Inhibitors or With Single-Agent Chemotherapy. JAMA Oncol 2018;4:1543-52.

11. Chiou VL, Burotto M. Pseudoprogression and ImmuneRelated Response in Solid Tumors. J Clin Oncol 2015;33:3541-3.

12. Wang Q, Gao J, Wu X. Pseudoprogression and hyperprogression after checkpoint blockade. Int Immunopharmacol 2018;58:125-35.

13. Fuentes-Antras J, Provencio M, Diaz-Rubio E. Hyperprogression as a distinct outcome after immunotherapy. Cancer Treat Rev 2018;70:16-21.

14. Hopkins AM, Rowland A, Kichenadasse G, et al. Predicting response and toxicity to immune checkpoint inhibitors using routinely available blood and clinical markers. Br J Cancer 2017;117:913-20.

15. Bagley SJ, Kothari S, Aggarwal C, et al. Pretreatment neutrophil-to-lymphocyte ratio as a marker of outcomes in nivolumab-treated patients with advanced non-small-cell lung cancer. Lung Cancer 2017;106:1-7.

16. Nakamura Y, Kitano S, Takahashi A, et al. Nivolumab for advanced melanoma: pretreatment prognostic factors and early outcome markers during therapy. Oncotarget 2016;7:77404-15.

17. Weber JS, Hodi FS, Wolchok JD, et al. Safety Profile of Nivolumab Monotherapy: A Pooled Analysis of Patients With Advanced Melanoma. J Clin Oncol 2017;35:785-92.

18. Weide B, Martens A, Hassel JC, et al. Baseline Biomarkers for Outcome of Melanoma Patients Treated with Pembrolizumab. Clin Cancer Res 2016;22:5487-96.

19. Bascioni R, Giorgi F. Waiting for an Answer: Hospice Care and Novel Immunotherapy. J Palliat Med 2015;18:1080-1.

20. Lee CK, Man J, Lord S, et al. Clinical and Molecular Characteristics Associated With Survival Among Patients Treated With Checkpoint Inhibitors for Advanced NonSmall Cell Lung Carcinoma: A Systematic Review and Meta-analysis. JAMA Oncol 2018;4:210-6.

21. Meng X, Huang Z, Teng F, et al. Predictive biomarkers in PD-1/PD-L1 checkpoint blockade immunotherapy. Cancer Treat Rev 2015;41:868-76.

22. Topalian SL, Taube JM, Anders RA, et al. Mechanismdriven biomarkers to guide immune checkpoint blockade in cancer therapy. Nat Rev Cancer 2016;16:275-87.

23. Toi Y, Sugawara S, Kawashima Y, et al. Association of Immune-Related Adverse Events with Clinical Benefit in Patients with Advanced Non-Small-Cell Lung Cancer Treated with Nivolumab. Oncologist 2018;23:1358-65.

24. Haratani K, Hayashi H, Chiba Y, et al. Association of Immune-Related Adverse Events With Nivolumab Efficacy in Non-Small-Cell Lung Cancer. JAMA Oncol 2018;4:374-8.

25. Brahmer JR, Lacchetti C, Schneider BJ, et al. Management of Immune-Related Adverse Events in Patients Treated With Immune Checkpoint Inhibitor Therapy: American Society of Clinical Oncology Clinical Practice Guideline. J Clin Oncol 2018;36:1714-68.

26. Haanen J, Carbonnel F, Robert C, et al. Management of toxicities from immunotherapy: ESMO Clinical Practice Guidelines for diagnosis, treatment and follow-up. Ann Oncol 2017;28:iv119-iv42.

27. Joshi MN, Whitelaw BC, Palomar MT, et al. Immune checkpoint inhibitor-related hypophysitis and endocrine dysfunction: clinical review. Clin Endocrinol (Oxf) 2016;85:331-9.

28. Gonzalez-Rodriguez E, Rodriguez-Abreu D. Immune Checkpoint Inhibitors: Review and Management of Endocrine Adverse Events. Oncologist 2016;21:804-16.

29. Abdel-Wahab N, Shah M, Suarez-Almazor ME. Adverse Events Associated with Immune Checkpoint Blockade in Patients with Cancer: A Systematic Review of Case Reports. PLoS One 2016;11:e0160221.

30. Suresh K, Naidoo J, Lin CT, et al. Immune Checkpoint Immunotherapy for Non-Small Cell Lung Cancer: Benefits and Pulmonary Toxicities. Chest 2018;154:1416-23.

31. Fujita K, Terashima T, Mio T. Anti-PD1 Antibody Treatment and the Development of Acute Pulmonary 
Tuberculosis. J Thorac Oncol 2016;11:2238-40.

32. Chu YC, Fang KC, Chen HC, et al. Pericardial Tamponade Caused by a Hypersensitivity Response to Tuberculosis Reactivation after Anti-PD-1 Treatment in a Patient with Advanced Pulmonary Adenocarcinoma. J Thorac Oncol 2017;12:e111-4.

33. Picchi H, Mateus C, Chouaid C, et al. Infectious complications associated with the use of immune checkpoint inhibitors in oncology: reactivation of tuberculosis after anti PD-1 treatment. Clin Microbiol Infect 2018;24:216-8.

34. Reynolds KL, Guidon AC. Diagnosis and Management of Immune Checkpoint Inhibitor-Associated Neurologic Toxicity: Illustrative Case and Review of the Literature. Oncologist 2019;24:435-43.

35. Lemiale V, Meert AP, Vincent F, et al. Severe toxicity from checkpoint protein inhibitors: What intensive care physicians need to know? Ann Intensive Care 2019;9:25.

36. Azoulay E, Shimabukuro-Vornhagen A, Darmon M, et al. Critical Care Management of Chimeric Antigen

Cite this article as: Lau KS, Liu R, Wong CC, Siu WKS, Yuen KK. Clinical outcome and toxicity for immunotherapy treatment in metastatic cancer patients. Ann Palliat Med 2020;9(6):4446-4457. doi: 10.21037/apm.2019.10.03
Receptor-T Cells-Related Toxicity: Be Aware and Prepared. Am J Respir Crit Care Med 2019;200:20-3.

37. Temel JS, Greer JA, Muzikansky A, et al. Early palliative care for patients with metastatic non-small-cell lung cancer. N Engl J Med 2010;363:733-42.

38. Hui D, Kim YJ, Park JC, et al. Integration of oncology and palliative care: a systematic review. Oncologist 2015;20:77-83.

39. Patil PD, Fernandez AP, Velcheti V, et al. Cases from the irAE Tumor Board: A Multidisciplinary Approach to a Patient Treated with Immune Checkpoint Blockade Who Presented with a New Rash. Oncologist 2019;24:4-8.

40. Khoja L, Day D, Wei-Wu Chen T, et al. Tumour- and class-specific patterns of immune-related adverse events of immune checkpoint inhibitors: a systematic review. Ann Oncol 2017;28:2377-85.

41. Zimmermann C, Swami N, Krzyzanowska M, et al. Early palliative care for patients with advanced cancer: a clusterrandomised controlled trial. Lancet 2014;383:1721-30. 